\begin{abstract}
This package of programs allows us to carry out relativistic calculations for many-electron atoms and ions. One can find energy levels and a number of atomic properties: $g$-factors, magnetic-dipole and electricquadrupole hyperfine structure constants, electric- and magnetic-multipole transition amplitudes, and matrix elements of the parity nonconserving interactions. Method of calculation is based on a combination of conventional configuration interaction (CI) method and many-body perturbation theory (MBPT). The former explicitly accounts for the interaction between valence electrons, while the latter includes core-core and core-valence correlations. These two methods are combined to acquire benefits from both approaches and attain better accuracy.
\end{abstract}

Keywords: Atomic structure, energy levels, matrix elements

\title{
PROGRAM SUMMARY
}

Manuscript Title: CI-MBPT: A package of programs for relativistic atomic calculations based on a method combining configuration interaction and many-body perturbation theory

Authors: M. G. Kozlov, S. G. Porsev, M. S. Safronova, I. I. Tupitsyn

Program Title: CI-MBPT

Journal Reference:

Catalogue identifier:

Licensing provisions: none

Programming language: Fortran 90

Computer: Intel Core i5 CPU, $3.2 \mathrm{GHz}$

Operating system: Linux (CentOS 5, Ubuntu 12.04 LTS, SUSE 13.2)

RAM: $8 \mathrm{~Gb}$

Keywords: Atomic structure, energy levels, matrix elements

Classification: 2.1

Nature of problem: Prediction of atomic or ionic energy levels and different observables in the framework of relativistic approach.

Solution method: The package of programs determines energy levels and associated many-electron wave functions for states of atoms and ions in the pure CI or CI+MBPT approximations. Using these wave functions, different atomic properties can be obtained, including $g$-factors, magnetic-dipole and electric-quadrupole hyperfine structure constants, electric- and magnetic-multipole transition amplitudes, P-odd and P,T-odd amplitudes.

\footnotetext{
* Corresponding author.

E-mail address: mgk@mf1309.spb.edu

** Corresponding author.

E-mail address: sporsev@gmail.com

Preprint submitted to Computer Physics Communications
}

April 28, 2015

(C) 2015. This manuscript version is made available under the Elsevier user license http://www.elsevier.com/open-access/userlicense/1.0/ 
Restrictions: The package is not designed for calculations of high Rydberg and autoionizing states. It becomes inefficient for the number of valence electrons exceeding four or five. It has not been tested for superheavy elements. The maximal number of Hartree-Fock-Dirac (HFD) orbitals when HFD equations are solved is 32.

Unusual features: One-electron orbitals outside the nucleus are defined on radial grid points. Inside the nucleus they are described in a form of the Taylor expansion over $r / R$, where $R$ is the nuclear radius.

Additional comments:

All programs have been compiled, linked, and tested with both "ifort" and freely available "gfortran".

Running time: Changes from tens of minutes for atoms with two valence electrons to tens of hours for more complex systems.

PACS: 2.70.-c; 32.10.-f; 31.15.ac

\section{Introduction}

In this write-up we describe a package of programs that can be applied for large-scale calculations of different atomic properties for the Dirac-Coulomb, or Dirac-Coulomb-Breit Hamiltonian in the no-pair approximation [1]. A main idea of our approach is to treat not only interaction between valence electrons but also include core-valence correlations. The former can be done in the framework of conventional configuration interaction (CI) method while for the latter we use many-body perturbation theory (MBPT). Since the CI method is known to be very successful for an explicit treatment of valence-valence interaction and the MBPT is very efficient in considering virtual core excitations, these two methods supplement each other quite well. Based on various calculations of different complexity of atomic properties, we conclude that a combination of CI and MBPT (below we refer to it as the CI+MBPT method) typically improves calculation accuracy by an order of magnitude in comparison to the pure valence CI.

There are several well-developed approaches to compute atomic structure and corresponding computer programs are available. In particular, there are atomic structure programs and packages by C. Froese Fischer and collaborators, such as the RELCI program for relativistic CI calculations [2] and the MCHF atomic structure package [3] based on dynamic memory allocation and sparse matrix methods. Indelicato and Desclaux designed the "Multiconfiguration Dirac-Fock and General Matrix Element" (MCDFGME) program for computation of the energy levels and different atomic properties [4, 5]. A general-purpose relativistic atomic structure GRASP package of Parpia, Froese Fisher, and Grant implementing many-configuration DiracHartree-Fock (MCDHF) approach [6], was further modified, extended and published in 2007 as GRASP2K [7]. New version of GRASP2K relativistic atomic structure package was recently published in [8].

Other generally applicable documented atomic structure codes include a program to calculate configuration interaction wave functions and electric-dipole oscillator strengths (CIV3) of A. Hibbert [9]; the atomic structure code (SUPERSTRUCTURE) of W. Eissner [10], yielding bound state energies in $L S$ coupling and intermediate coupling as well as associated radiative data; the COWAN code 11], an integrated computer package for atomic processes in plasmas (HULLAC) 12], the ATOM package 13] and the flexible atomic code (FAC) [14]. Relativistic multireference Møller-Plesset perturbation theory was developed and implemented with analytic basis sets of Gaussian spinors in [15].

The common problem of the MCHF and CI methods is that the number of configurations for a manyelectron atom depends exponentially on the number of correlated electrons. Therefore, it is possible to include only a small fraction of them for a system with a few (more than 4, or 5) electrons. Usually it is done by dividing all electrons into the core and valence electrons and neglecting core excitations. This severely limits the accuracy of these methods when they are applied to heavy many-electron atoms. A way to circumvent this problem was suggested by Dzuba, Flambaum, and Kozlov in 1996 [16]. They exploited the idea that the CI and MBPT methods deal with different sectors of the many-body problem. CI explicitly treats interactions between several valence electrons, while MBPT can be effectively used to account for 
core-core and core-valence correlations. For this reason two methods can be combined to acquire benefits from both approaches and attain higher accuracy. Note that somewhat different variant of the combination of CI with MBPT was suggested by Savukov and Johnson [17]. Another program package also implementing ideas of Ref. [16] was used by Dzuba and co-authors (see, for example, [18, 19] and references therein).

The CI+MBPT method was initially designed for accurate calculations of energy levels of atoms and ions [16]. During last two decades the method was developed, modified, and extended. At present it also allows for calculating different atomic observables such as hyperfine structure constants, oscillators strengths, lifetimes, parity-nonconserving amplitudes, etc. The method was extensively used for calculating the properties of atoms and ions of different complexity (see, e.g., 20-25]). For example, the different properties of atomic thallium, considered as the three-valence atom (with 78 core electrons), were computed with the accuracy $2-3 \%$ 24, 25].

In certain cases the CI+MBPT method becomes less effective. The accuracy of the method deteriorates when the energy gap between core and valence shells is too small (typically it should be larger than the excitation energy for the highest valence state considered). When the number of valence electrons becomes too large $(\geq 5)$ the CI+MBPT method becomes impractical. Due to the enormous size of the CI space it is impossible to saturate CI even for a short basis set. Moreover, for such a large number of valence electrons the effective Hamiltonian can no longer be accurately represented by a two-electron operator. The three-particle MBPT corrections [16] become quite important and should be included into consideration leading to the Hamiltonian matrix that is much less sparse than for the pure CI.

It is worth noting that the CI part of the package is completely independent from the MBPT part. All calculations can be carried out in the framework of the pure CI method, disregarding MBPT corrections. The accuracy in this case is typically an order of magnitude lower, but such an approach gives an additional flexibility. For example, it is possible to use the basis set of non-Dirac-Fock orbitals. This allows us to significantly reduce the size of the CI space without decreasing the accuracy. This is particularly important for the large number of valence electrons.

Generally, the calculation process consists of the following stages:

- Numerical solution of the Hartree-Fock-Dirac (HFD) equations using finite difference method. We find single-electron HFD energies and wave functions for the core and valence electrons.

- Construction of virtual orbitals. Together with the HFD orbitals they form basis set for the CI and MBPT calculations. Note that it corresponds only to the positive Dirac spectrum.

- Calculation of one-electron and two-electron radial integrals for valence electrons.

- Calculation of one- and two-electron matrix elements of the correlation potential $\Sigma$ in the framework of the second order MBPT. This step allows us to account for the core-valence correlations and can be skipped for the pure CI calculation.

- Carrying out relativistic CI calculation (with or without $\Sigma$ corrections) and obtaining the eigenvalues and eigenvectors of the multi-particle Schrödinger equation.

- Calculation of different atomic properties using the wave functions found in the previous step.

\section{Theory}

The CI+MBPT method is described in detail in [16, 26, 27]. In this section we only briefly outline its main features. All electrons are divided into two groups, the core and valence electrons. The effective Hamiltonian for the valence electrons is formed using the MBPT technique for the interaction of the valence electrons with the core. CI method is used then to find the energy levels and the wave functions of the atom. Configurations with excitations from the core are not explicitly included at the CI stage of the calculations.

The Hilbert space of the many-electron problem is divided into two subspaces. The first subspace $P$ corresponds to the frozen-core approximation. The second subspace $Q$, including core excitations, is 
complimentary to the first one, so that $P+Q=1$. The $P$ subspace is infinite-dimensional, so one can not find exact solution of the Schrödinger equation. However, we can approximate the subspace $\mathrm{P}$ by a finite-dimensional configurational space $P_{\mathrm{CI}} \subset P$ in the framework of the CI method. We assume that the $P_{\mathrm{CI}}$ subspace can be chosen so that the required accuracy of the solution of the Schrödinger equation can be achieved and we do not distinguish between $P_{\mathrm{CI}}$ and $P$ subspaces in the text below.

The many-electron wave function from the $P$ subspace is presented as a linear combination of Slater determinants,

$$
\Phi=\sum_{\operatorname{det}_{I} \in P} C_{I}\left|\operatorname{det}_{I}\right\rangle
$$

Varying the coefficients $C_{I}$, we arrive at the matrix eigenvalue problem:

$$
\sum_{\operatorname{det}_{I} \in P}\left\langle\operatorname{det}_{J}|H| \operatorname{det}_{I}\right\rangle C_{I}=E C_{J} .
$$

The CI Hamiltonian can be written as

$$
H_{\mathrm{CI}}=P H P
$$

or, in explicit form, as

$$
H_{\mathrm{CI}}=E_{\text {core }}+\sum_{i>N_{\text {core }}} h_{i}^{\mathrm{CI}}+\sum_{j>i>N_{\text {core }}} V_{i j},
$$

where $N_{\text {core }}$ is the number of core electrons, $E_{\text {core }}$ is the energy of the core which includes kinetic energy of the core electrons, Coulomb energy of their interaction with the nucleus and potential energy of the corecore electrostatic interaction, but not the core-valence interaction. The latter is included in the one-electron operators $h_{i}^{\mathrm{CI}}$ together with the kinetic energy of the valence electrons and their interaction with the nucleus. The last term in Eq. (4) accounts for the valence-valence interaction.

In the framework of the pure CI method we solve the eigenvalue problem $H_{\mathrm{CI}} \Phi=E_{\mathrm{CI}} \Phi$ in the basis set of Slater determinants (2). This equation is approximate as it ignores the subspace $Q$. To write the exact equivalent of the original Schrödinger equation in the subspace $P$, we can make $P, Q$-decomposition of the Hamiltonian and the wave function of the many body problem. Taking into account that $P+Q=1$, we have

$$
\begin{aligned}
H & =H_{\mathrm{CI}}+P H Q+Q H P+Q H Q \\
\Psi & =P \Psi+Q \Psi \equiv \Phi+\chi
\end{aligned}
$$

The Schrödinger equation

$$
H \Psi=E \Psi
$$

can be written as a system of equations for $\Phi$ and $\chi$ and then $\chi$ can be expressed via $\Phi$. After some transformations we obtain Schrödinger-like equation in $P$ subspace with the energy-dependent effective Hamiltonian [16]:

$$
\begin{aligned}
& H_{\mathrm{eff}} \equiv H_{\mathrm{CI}}+\Sigma(E) \\
& H_{\mathrm{eff}} \Phi=E \Phi, \\
& \Sigma(E)=(P H Q) R_{Q}(E)(Q H P),
\end{aligned}
$$

where $R_{Q}(E)$ is the Green's function in $Q$ subspace defined as

$$
R_{Q}(E)=Q(E-H)^{-1} Q .
$$



as

$$
\left\langle\Phi_{i}\left|1+(P H Q) R_{Q}\left(E_{i}\right) R_{Q}\left(E_{k}\right)(Q H P)\right| \Phi_{k}\right\rangle=\delta_{i k} .
$$

Equations (9) -(11) are exact equivalent of Eq. (7). Note that Eq. (11) can be simplified if we assume that $E_{i} \simeq E_{k} \equiv E_{\mathrm{av}}$. It can be done if we are interested only in sufficiently low-lying energy levels. In this approximation Eq. (11) is expressed through the derivative of the operator $\Sigma$ as

$$
\left\langle\Phi_{i}\left|1-\partial_{E} \Sigma(E)\right| \Phi_{k}\right\rangle_{E=E_{\mathrm{av}}}=\delta_{i k} .
$$

For a properly chosen subspace $P$, the derivative $\partial_{E} \Sigma(E)$ can be negligibly small and usual orthonormality condition can be applied [16]. In this case, Eq. (9) can be solved using the standard CI approach, provided that the operator $\Sigma\left(E_{\mathrm{av}}\right)$ is calculated beforehand. The latter can be done with the help of the many-body perturbation theory.

If we define $N_{\mathrm{DF}}$ as the number of electrons included in Dirac-Fock self-consistent procedure: $N_{\text {core }} \leq$ $N_{\text {DF }} \leq N$, where $N$ is the number of electrons in the atom, then the matrix elements of the operator $\Sigma$ in the CI space, $\Sigma_{I J} \equiv\left\langle\operatorname{det}_{I}|\Sigma| \operatorname{det}_{J}\right\rangle$, can be written as [16]

$$
\begin{aligned}
\Sigma_{I J}(E)= & \sum_{M \in Q} \frac{U_{I M} U_{M J}}{E-E_{M}}+ \\
& \sum_{M, L \in Q} \frac{U_{I M} U_{M L} U_{L J}}{\left(E-E_{M}\right)\left(E-E_{L}\right)}+\ldots \\
\equiv & \Sigma^{(2)}+\Sigma^{(3)}+\ldots
\end{aligned}
$$

Here the operator $U$ is the residual interaction determined as $U=V-V^{N_{\mathrm{DF}}}$, where $V$ is the operator of the two-electron Coulomb-, or Coulomb-Breit interaction and $V^{N_{\mathrm{DF}}}$ is the interaction with the Dirac-Fock field of the $N_{\mathrm{DF}}$ electrons.

If we restrict ourselves by including $\Sigma$ in the lowest (second-) order, we arrive at the matrix equation of the combined CI and MBPT method [16]

$$
\sum_{J \in P}\left(H_{I J}+\sum_{M \in Q} \frac{U_{I M} U_{M J}}{E-E_{M}}\right) C_{J}=E C_{I} .
$$

Generally speaking the second term in the brackets is a three-electron operator. However, the one-electron and two-electron parts of this term dominate for the atoms with the small number of valence electrons $N_{v}$. For the combinatorial reasons the three-electron part grows much faster with the number of the valence electrons and may become important for $N_{v}>4$. In the present implementation of the package three-electron term of the effective Hamiltonian is neglected. The one-electron and two-electron parts of the operator $\Sigma$ are calculated using MBPT. Each contribution is represented by a number of the Brueckner-Goldstone diagrams given in [16] and is stored in the same way as one- and two-electron radial integrals.

\section{Overview of the package}

The package consists of 10 programs which are listed in Table1 and shown in the block-scheme in Fig. 1 For each program there is a source fortran file with the same name. All necessary subroutines are included via fortran command include. Respective files have extensions inc and should be in the same directory as the main files. We do not use dynamic memory allocation and all array dimensions are defined by parameters. Parameter files have extension par and also should be in the same directory. Short comments defining the parameters and rough estimates of relevant memory scaling are included in parameter files. The file phys . par includes physical parameters such as the speed of light, Rydberg constant, and so on. We 
Table 1: List of the CI-MBPT package programs. In columns "Name" and "Main file" we give the name of the program and the main fortran file. Array dimensions are defined in the parameter files listed in the column "Param.". Text input files are given in the column "Input". Required binary files are listed in the column "Using". Formed binary files are presented in the column "Forming" (the file SGC.CON is an ASCII file). The ASCII files with results are listed in last column. The files given in the parenthesis are used only in some regimes.

\begin{tabular}{|c|c|c|c|c|c|c|}
\hline $\begin{array}{c}\text { Name } \\
\text { (Main file) }\end{array}$ & Description & Param. & Input & Using & Forming & Results \\
\hline $\begin{array}{c}\text { hfd } \\
\text { (hfd.for) }\end{array}$ & $\begin{array}{c}\text { Solves HFD } \\
\text { equations }\end{array}$ & $\begin{array}{l}\text { hfd.par } \\
\text { phys.par }\end{array}$ & HFD.INP & & HFD.DAT & HFD.RES \\
\hline $\begin{array}{c}\text { bass } \\
\text { (bass.for) }\end{array}$ & $\begin{array}{c}\text { Constructs } \\
\text { basis set }\end{array}$ & $\begin{array}{r}\text { hfd.par } \\
\text { conf.par } \\
\text { bspl.par } \\
\text { phys.par }\end{array}$ & BASS.INP & HFD.DAT & HFD.DAT & BASS.RES \\
\hline $\begin{array}{c}\text { add } \\
\text { (add.for) }\end{array}$ & $\begin{array}{l}\text { Constructs } \\
\text { input file }\end{array}$ & $\begin{array}{l}\text { add.par } \\
\text { conf.par }\end{array}$ & ADD.INP & & CONF.INP & \\
\hline $\begin{array}{c}\text { basc } \\
\text { (basc.for) }\end{array}$ & $\begin{array}{l}\text { Calculates } \\
\text { radial } \\
\text { integrals }\end{array}$ & $\begin{array}{r}\text { hfd.par } \\
\text { conf.par } \\
\text { phys.par }\end{array}$ & CONF.INP & HFD.DAT & $\begin{array}{l}\text { CONF.DAT } \\
\text { CONF.GNT } \\
\text { CONF.INT }\end{array}$ & BASC.RES \\
\hline $\begin{array}{c}\text { sgc } \\
\text { (sgc.for) }\end{array}$ & $\begin{array}{c}\text { Calculates } \\
\text { one-electron } \\
\text { MBPT diagrams }\end{array}$ & $\begin{array}{r}\text { hfd.par } \\
\text { conf.par } \\
\text { phys.par }\end{array}$ & MBPT.INP & $\begin{array}{l}\text { HFD.DAT } \\
\text { CONF.DAT }\end{array}$ & SGC.CON & \\
\hline $\begin{array}{c}\text { scrc } \\
\text { (scrc.for) }\end{array}$ & $\begin{array}{c}\text { Calculates } \\
\text { two-electron } \\
\text { MBPT diagrams }\end{array}$ & $\begin{array}{r}\text { hfd.par } \\
\text { conf.par } \\
\text { phys.par }\end{array}$ & MBPT.INP & $\begin{array}{l}\text { HFD.DAT } \\
\text { CONF.DAT }\end{array}$ & SCRC.CON & SCRC.RES \\
\hline $\begin{array}{c}\text { conf } \\
\text { (conf.for) }\end{array}$ & $\begin{array}{l}\text { Carries out } \\
\text { CI or } \\
\text { CI+MBPT } \\
\text { calculations }\end{array}$ & $\begin{array}{r}\text { hfd.par } \\
\text { conf.par } \\
\text { phys.par } \\
\text { hread.par }\end{array}$ & CONF.INP & $\begin{array}{c}\text { CONF.DAT } \\
\text { CONF.GNT } \\
\text { CONF.INT } \\
\text { (SGC.CON) } \\
\text { (SCRC.CON) }\end{array}$ & $\begin{array}{c}\text { CONF.DET } \\
\text { CONF.HIJ } \\
\text { CONF.XIJ } \\
\text { CONF.JJJ }\end{array}$ & CONF.RES \\
\hline $\begin{array}{c}\mathrm{dtm} \\
(\mathrm{dtm} . \text { for })\end{array}$ & $\begin{array}{l}\text { Calculates hfs } \\
\text { constants, } M k \\
\& E k \text { transition } \\
\text { amplitudes, etc }\end{array}$ & $\begin{array}{r}\text { hfd.par } \\
\text { conf.par } \\
\text { phys.par }\end{array}$ & CONF.INP & $\begin{array}{c}\text { CONF.DAT } \\
\text { CONF.DET } \\
\text { CONF.XIJ } \\
\text { (CONF1.XIJ) } \\
\text { (CONF1.DET) }\end{array}$ & DTM.INT & $\begin{array}{c}\text { (DM.RES) } \\
\text { or } \\
\text { (TM.RES) }\end{array}$ \\
\hline $\begin{array}{c}\text { rpa } \\
\text { (rpa.for) }\end{array}$ & $\begin{array}{l}\text { Calculates } \\
\mathrm{RPA}^{\mathrm{b}} \\
\text { corrections }\end{array}$ & $\begin{array}{r}\text { rpa.par } \\
\text { hfd.par } \\
\text { phys.par }\end{array}$ & MBPT.INP & $\begin{array}{l}\text { HFD.DAT } \\
\text { CONF.DAT }\end{array}$ & RPA_n.INT ${ }^{\mathrm{a}}$ & RPA.RES \\
\hline $\begin{array}{c}\text { rpa_dtm } \\
\text { (rpa_dtm.for) }\end{array}$ & $\begin{array}{l}\text { Writes RPA } \\
\text { corrections } \\
\text { to DTM.INT }\end{array}$ & $\begin{array}{l}\text { rpa.par } \\
\text { conf.par }\end{array}$ & & $\begin{array}{l}\text { DTM.INT } \\
\text { RPA_n.INT }\end{array}$ & DTM.INT & RPA_DTM.RES \\
\hline
\end{tabular}

$\overline{\bar{a}}$ Here the number $n=1-13$ numerates one-electron operators for which RPA corrections are calculated (see Sec. 5.3 for more detail). For $n \geq 10$ the notation RPAn.INT is used;

${ }^{\mathrm{b}}$ Random phase approximation. 
expect that the fortran codes will be used as they are, but the parameter files can be adjusted for the needs of the user.

All programs require text input files NAME. INP, where name refers to the name of the corresponding program (see Table 1). Sometimes several programs use the same input file. These files typically consist of three parts. The first part includes (small) number of required parameters, such as the nuclear charge $\mathrm{Z}$, the number of core- and valence electrons, Nso and Ne, etc. The second part includes optional parameters and may be omitted. The programs use default values for such omitted inputs. The third part includes a (possibly long) list of either orbitals, or configurations. The file MBPT. INP consists of several independent blocks. Each of them starts with the respective label. The structure of each block is fixed, but their order is arbitrary.

Several programs also require an interactive input in the dialogue regime. The input dialog is usually very short and allows us to choose between few options. The main results of the calculations are displayed on the screen and a more detailed output is saved to the files NAME. RES, where the NAME, as a rule, is the same as the name of the program.

An advantage of our package is that it does not require any programs from an external library. All required subroutines are provided with the package. Compilation is easy if all files of the package (*.for, $*$.inc, and *.par) are in the same directory. If we use the Intel Fortran (ifort), we can make executable file, for example, for the program hfd, with the simple command ifort -o hfd hfd.for. Additional optimization, or debugging options can be added in a standard way.

Below we describe each program of the package in more detail. We start with programs which are necessary for the CI calculations. They can be used without, or with the MBPT part of the package, which will be described later. Finally, in the Appendix we give a very short description of the inc files.

\section{Description of programs for the CI calculations}

Here we describe the programs required for the CI calculations in more detail and give a description of required parts of the input files. Only the most important optional parameters are discussed and the other ones are listed in the file inpstr.inc with short comments.

\section{1. $\mathrm{hfd}$}

The name of this program comes from the first letters of Hartree, Fock, and Dirac. The hfd program solves restricted Hartree-Fock-Dirac (HFD) equations self-consistently and finds two-component Dirac-Fock orbitals and eigenvalues of the HFD Hamiltonian. In the restricted HFD method the orbitals are found in the central field approximation and depend only on principal, orbital, and total angular momentum quantum numbers $n, l$, and $j$. An initial version of this code was described in [28]. Though the code was evolving during the years the main features remained unchanged. An example of the file HFD. INP for the neutral Magnesium looks as follows: 


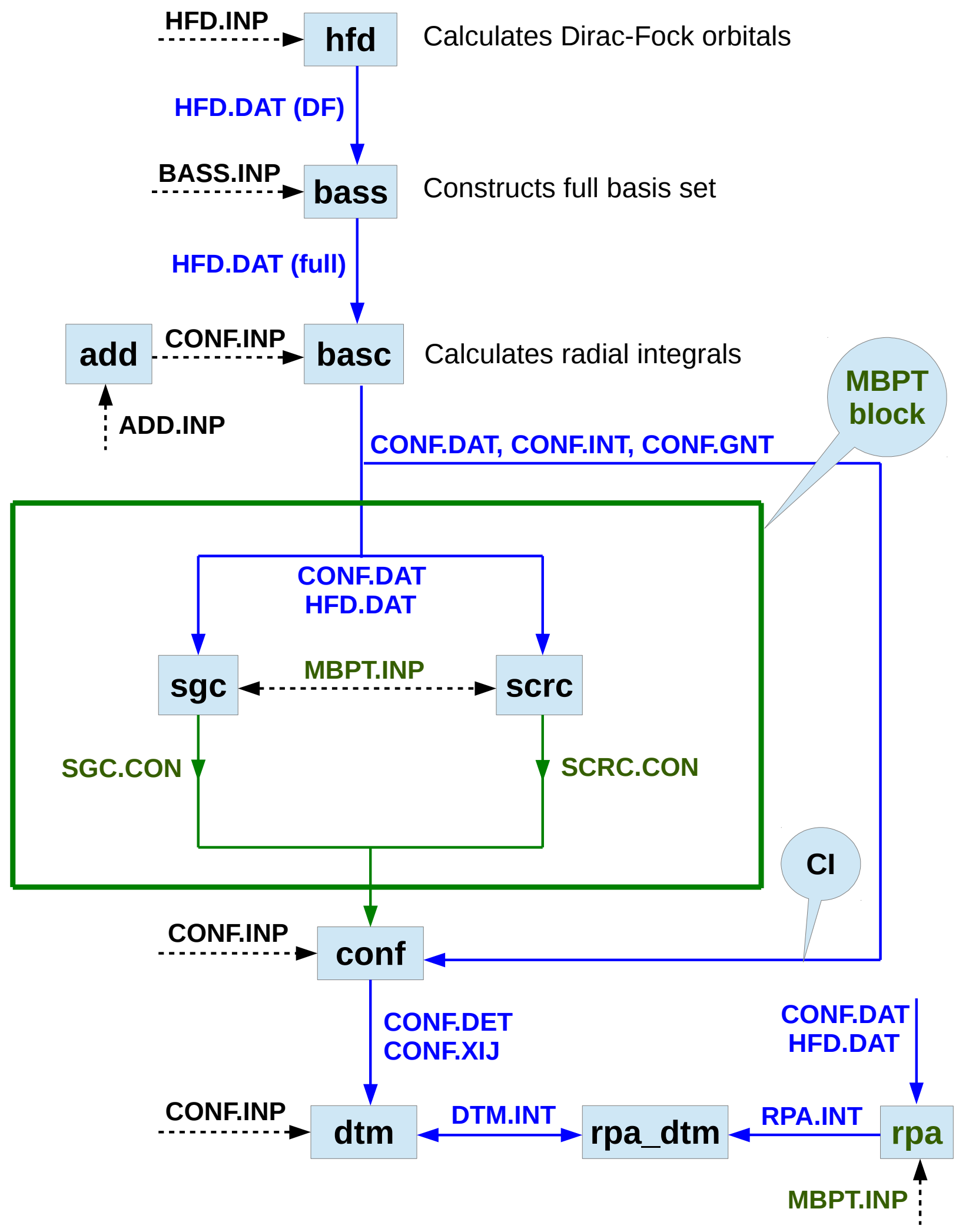

Figure 1: (Color online) Block-scheme of the program package. Required input ASCII files are given by dashed arrows (black online). Solid arrows correspond to the input/output binary files (blue online). 


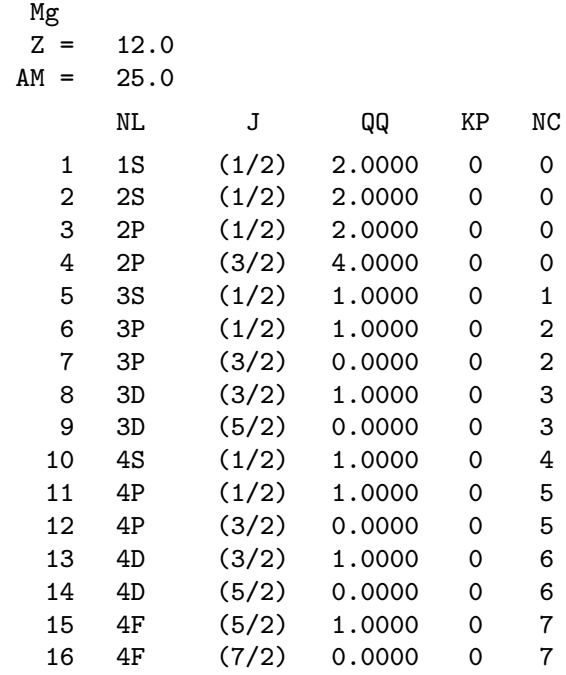

The first three lines define the name of the element, its nuclear charge $\mathbf{Z}$, and the total number of nucleons Am. If $A m<0$ the nucleus is pointlike, otherwise the nucleus is the uniformly charged ball, which radius depends on Am. The columns labeled NL, J, and QQ define the principal and the orbital quantum numbers, the total angular momentum of the relativistic shell, and the number of electrons in the shell, respectively.

The key KP=0 means that the orbital is found from the HFD equations and written to the file HFD.DAT. The key KP=1 means that the orbital is taken from the file HFD.DAT and kept frozen. With the help of this key it is possible to form basis sets of orbitals, which correspond to the different Hartree-Fock potentials.

The column NC defines configuration. The HFD equations are solved for each configuration separately. First, the orbitals for the configuration $\mathrm{N} c=0$ are found. Then they are frozen and the orbitals for $\mathrm{N} c=1$ are found, etc. The electrons belonging to the same configuration interact with each other and with the Dirac-Fock field of the electrons from zeroth configuration. In our example each configuration with Nc>0 includes only one electron, so all electrons are in the same field, which corresponds to the configuration $\left[1 s^{2} 2 s^{2} 2 p^{6}\right]$. Therefore, this input file corresponds to the $V^{N-2}$ approximation, where $N$ is the total number of electrons and number of the electrons included in the zeroth configuration is $N-2$.

If we want to form the basis set in the $V^{N}$ approximation, we need to include both $3 s$ electrons in the zeroth configuration and change respective occupation number to 2 . Otherwise the input remains unchanged.

The hfd program determine orbitals from an expression for the average energy of a nonrelativistic configuraton, redefining the occupation numbers $\mathrm{QQ}_{l, j}$ of relativistic shells with $l>0$ given in HFD. INP (where $j$ and $l$ are the total and orbital angular momenta of the shell) as $\mathrm{q}_{l j}=\left(\mathbf{Q Q}_{l, j=l-1 / 2}+\mathbf{Q Q}_{l, j=l+1 / 2}\right) *$ $(2 j+1) /(4 l+2)$. In the example above, there is one electron in the configuration $\mathrm{Nc}=3$. It will be redistributed between two relativistic shells with the weights $Q Q=0.4$ for the $3 d_{3 / 2}$ shell and $Q Q=0.6$ for the $3 d_{5 / 2}$ shell. Thus, the occupation number can be fractional.

Below we illustrate how several optional keys can be added to the head of HFD. INP, if it is needed.

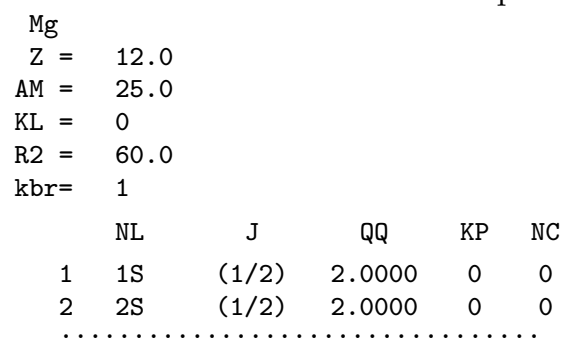

\begin{tabular}{|c|c|c|c|c|}
\hline & NL & $\mathrm{J}$ & QQ & KP \\
\hline 1 & 1S & $(1 / 2)$ & 2.0000 & 0 \\
\hline 2 & $2 \mathrm{~S}$ & $(1 / 2)$ & 2.0000 & 0 \\
\hline
\end{tabular}

The key KL defines initial approximation. The default value is $\mathrm{KL}=0$.

- $\mathrm{KL}=0$ - calculation starts from the beginning using standard initial approximation. 
- $\mathrm{KL}=1$ - continuation of the previous calculation (used when self-consistency has not been reached). The file HFD.DAT is required. All parameters should be unchanged.

- $\mathrm{KL}=2$ - initial approximation is taken from the file HFD.DAT.

- $\mathrm{KL}=3$ - all orbitals in the initial approximation are put to be zero.

Parameter R2 - is the last point of the radial grid in a.u. The default value is $\mathrm{R} 2=50.0$. The key $\mathrm{kbr}=$ 0,1 . The default value is $\mathrm{kbr}=0$. For $\mathrm{kbr}=1-$ the magnetic part of the Breit interaction (the Gaunt term) is included.

\section{2. bass}

The program bass is used for construction of the basis set. Typically we start with forming Dirac-Fock orbitals for the core shells and the valence shells, which are occupied in the atomic states we are interested in. Then we add virtual orbitals to account for correlations. It can be done in many different ways. Here we briefly describe the simple, but rather efficient way of constructing virtual orbitals, suggested first by $\mathrm{P}$. Bogdanovich and G. Ẑukauskas in [29, 30] and further developed by our group (see, e.g., [31]).

A reasonable basis set should consist of the orbitals mainly localized at the same distances from the origin as the valence orbitals. To avoid the problem with intruder states and to calculate such observables, as the hyperfine structure constants and parity-nonconserving amplitudes, these orbitals should have proper behavior near the origin $\left(\sim r^{\gamma_{j}}\right.$ for the point-like nucleus, where $\gamma_{j}=\sqrt{(j+1 / 2)^{2}-(\alpha Z)^{2}}, j$ is the total angular momentum of the orbital, and $\alpha$ is the fine-structure constant). To meet these conditions we can take a previously constructed orbital, $f_{\text {old }}$, and multiply it by a function $\phi$, which tends to zero at the origin and is not growing too fast at large distances. The lowest virtual orbitals we construct from the HFD orbitals. After orthogonalization we get an orbital with the proper behavior near the nucleus and at the infinity. One can choose $\phi=r$ or $\phi=\sin (k r)$, and determine new orbital $f_{\text {new }}$ as

$$
f_{\text {new }}=r^{\Delta l} \phi f_{\text {old }} \text {. }
$$

Here $\Delta l=l_{\text {new }}-l_{\text {old }}$, where $l_{\text {new }}$ and $l_{\text {old }}$ are the orbital quantum numbers and $l_{\text {new }}>l_{\text {old }}$. For $l_{\text {new }} \leq l_{\text {old }}$, $\Delta l$ is taken to be 0 .

To avoid an appearance of intruder states, we apply this procedure only to the large component $f_{n l j}$ of the radial Dirac bispinor. The small component $g_{n l j}$ is found from the kinetic balance condition:

$$
g_{n l j}=\frac{\boldsymbol{\sigma} \boldsymbol{p}}{2 m c} f_{n l j},
$$

where $\boldsymbol{\sigma}=\left(\sigma_{x}, \sigma_{y}, \sigma_{z}\right)$ are the Pauli matrices, $\boldsymbol{p}$ and $m$ are the electron momentum and mass, and $c$ is the speed of light. The newly constructed functions are then orthonormalized with respect to the functions of the same symmetry. An advantage of this approach is that it requires very few input data and the basis set can be constructed automatically.

Other options of construction of the basis set are also implemented. In particular, the bass program can construct large components of the orbitals from $B$-splines, or take them from an external file. Small components are still formed with the kinetic balance method. One can also combine all three methods. Unfortunately, these variants require certain manipulations and experimentation with the file BASS.INP. For this reason they are more difficult for users, who just start using the package. We recommend for the beginners to use the "auto" regime (see below).

The program bass starts from reading the input file BASS. INP. An example of this file for Mg I is given below. 


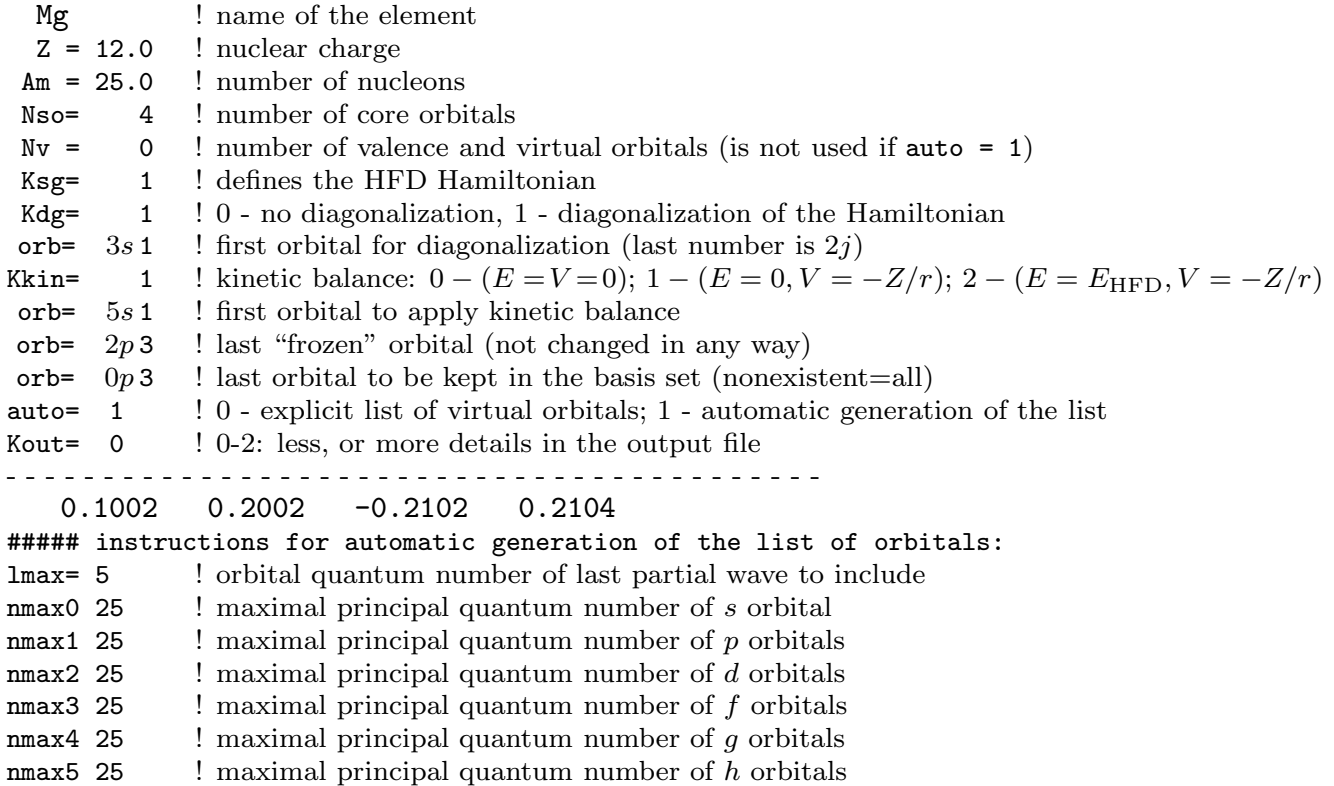

Let us discuss the keys which are important for "auto" regime, i.e. for auto=1. Nv is the total number of valence and virtual orbitals. In the regime "auto" it is determined automatically. Kdg $=1$ means that after construction of the virtual orbitals the Hamiltonian is diagonalized. This has to be done for CI+MBPT; for pure CI one can use $\mathrm{Kdg}=0$. The key $\mathrm{Ksg}=1$ means diagonalization of the HFD Hamiltonian and forming Dirac-Fock orbitals. The first orbital for diagonalization is typically the first orbital above the core. The first orbital to apply kinetic balance is typically the first virtual (non Dirac-Fock) orbital. The key Kkin $=0,1,2$ allows us to add extra terms to the kinetic balance equation (16). The optional key Kout $=0,1,2$ determines level of details in the output file BASS.RES.

The decimal numbers below the dashed line give the quantum numbers $n, l, j$ and the occupation numbers $q$ of the Nso core shells. Let us consider as an example the number -0.2102 . The principal quantum number $n$ is determined as $n=\operatorname{INT}(10 \times 0.2102)=2$ (where INT is the integer part of the number). The orbital quantum number $l$ is given by the second decimal digit in the number -0.2102 , i.e., $l=1$. Finally two last decimal digits give the occupation number $q$. In our example $q=2$. The sign \pm in front of the number correspond to the total angular momentum $j=l \pm 1 / 2$. Thus, the number -0.2102 corresponds to $2 p_{1 / 2}^{2}$ and the number 0.2104 corresponds to $2 p_{3 / 2}^{4}$. Similarly, 0.1002 means $1 s_{1 / 2}^{2}$ and 1.4203 corresponds to $14 d_{5 / 2}^{3}$.

In total, the numbers $0.1002,0.2002,-0.2102,0.2104$ below the dashed line specify the core shells of the atom. In our case of atomic $\mathrm{Mg}$, there are four relativistic core shells. If the number of relativistic core shells exceeds 6 , the 7 -th core shell should start from the same position of the next line.

In the last part of the file the parameter lmax is the maximal orbital quantum number of the partial wave which will be constructed. The total number of the (nonrelativistic) partial waves is $1 \max +1$. For example, $\operatorname{lmax}=5$ means that $s, p, d, f, g$ and $h$ orbitals will be constructed. The keys nmax 0, nmax 1 , etc, determine maximum principal quantum number of the orbital in each partial wave. In our Mg example, after running the hfd and bass programs with the input files HFD. INP and BASS. INP discussed above, we get the basis set for $\mathrm{Mg}[25 s 25 p 25 d 25 f 25 \mathrm{~g} 25 \mathrm{~h}]$ constructed in the $V^{N-2}$ approximation.

\section{3. add}

The add program forms the input file CONF. INP subsequently used by the programs basc, conf, and $\mathrm{dtm}$. This file includes the list of (relativistic) configurations, which define the CI space. We construct configurations by exciting electrons from the reference configuration(s). In Fig. 2 we give an example of the input file ADD. INP used for construction of the odd-parity configurations of Mg. 


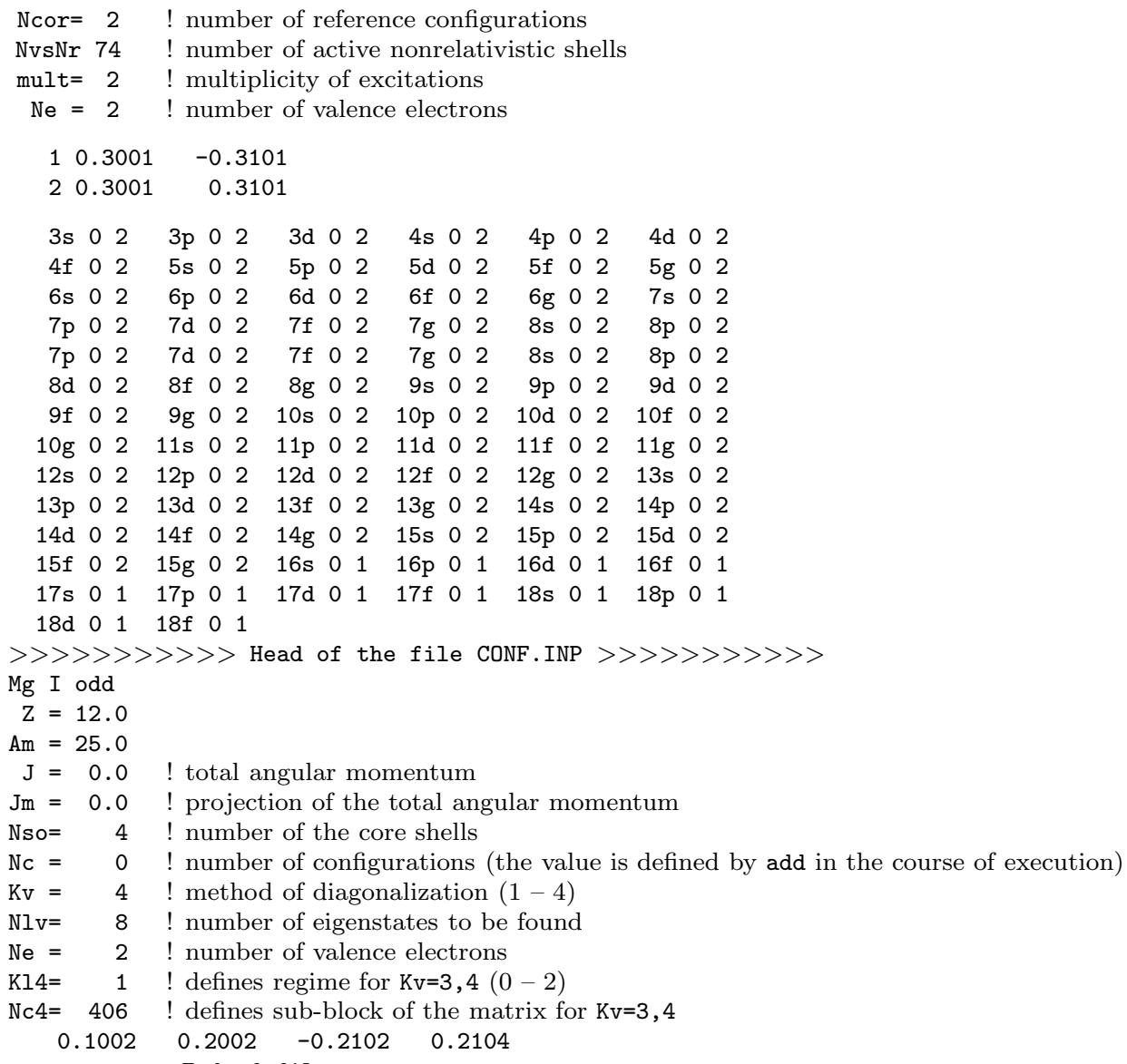

Figure 2: The file ADD. INP for the odd-parity states of Mg.

This input file specifies Ncor $=2$ reference configurations, namely, $3 s 3 p_{1 / 2}$ and $3 s 3 p_{3 / 2}$ presented in the same format as the core shells in the file BASS. INP:

$$
\begin{array}{rrr}
1 & 0.3001 & -0.3101 \\
2 & 0.3001 & 0.3101
\end{array}
$$

The add program performs excitations of the electrons from the reference configurations to the $\mathrm{NvsNr}=74$ 'active' nonrelativistic shells. The multiplicity of excitations is defined by the parameter mult, i.e., in this example single and double excitations are done. For each of the 74 nonrelativistic shells we define minimal and maximal occupation numbers. For example, if we put $7 \mathrm{~g} 01$ instead of $7 \mathrm{~g} 02$, then only single excitations to the $7 g$ shell are allowed. Similarly, if we put 3s 12 , the minimal occupation number of the $3 s$ shell is 1 . In our example it means that no excitations from this shell are allowed. The second part of the file ADD. INP defines the head of the file CONF. INP. We describe this file in more detail in the Section 4.5.

Remark 1: The order of shells in configurations and in the list of active shells should be exactly the same as the order of orbitals in the file HFD.DAT. The full list of the orbitals in the basis set is given in the file BASS.RES.

Remark 2: As long as we are doing single and double excitations for the two-valence system, it is sufficient to give a single main reference configuration to form a complete list of configurations. However, it is preferable to include all relativistic configurations, which belong to the same nonrelativistic configuration. 
Program basc calculates one-electron and two-electron radial integrals, which are used by the program conf to form the matrix of the Hamiltonian in the CI space. One-electron radial integrals correspond to the Hartree-Fock potential of the core. Two-electron radial integrals account for the Coulomb, and (optionally) magnetic part of the Breit interaction between valence electrons. The matrix element of the Coulomb interaction for the multipolarity $k$ can be written as:

$$
\left\langle c, d\left|V_{q}^{k}\right| a, b\right\rangle \equiv G_{q}^{k}(c a) G_{q}^{k}(b d) R_{a b c d}^{k},
$$

where the angular factors $G_{q}^{k}(f i)$ (known as relativistic Gaunt coefficients) are given by

$$
G_{q}^{k}(f i)=(-1)^{m_{f}+1 / 2} \delta_{p} \sqrt{\left(2 j_{i}+1\right)\left(2 j_{f}+1\right)}\left(\begin{array}{ccc}
j_{f} & j_{i} & k \\
-m_{f} & m_{i} & q
\end{array}\right)\left(\begin{array}{ccc}
j_{f} & j_{i} & k \\
1 / 2 & -1 / 2 & 0
\end{array}\right),
$$

$R_{a b c d}^{k}$ are the relativistic Coulomb radial integrals, and $\delta_{p}$ accounts for the parity selection rule:

$$
\delta_{p}=\xi\left(l_{i}+l_{f}+k\right), \quad \xi(n)= \begin{cases}1 & \text { if } n \text { is even } \\ 0 & \text { if } n \text { is odd }\end{cases}
$$

The Breit interaction has the same form (17), but without parity selection rule (19).

The basc program reads the file CONF. INP to determine which radial integrals are needed. It calculates these integrals and writes them to the file CONF. INT. The angular factors (18) are saved to the file CONF. GNT. This program also forms the file CONF. DAT which is similar to the file HFD.DAT. Both files include basis radial orbitals $\phi_{n l j}$ :

$$
\phi_{n l j} \equiv r\left(\begin{array}{r}
f_{n l j} \\
-g_{n l j}
\end{array}\right) .
$$

The file HFD . DAT also includes radial derivatives of the orbitals $\partial_{r} \phi_{n l j}$, while the file CONF. DAT stores functions $\chi_{n l j}=h_{\mathrm{DF}}^{\mathrm{r}} \phi_{n l j}$, where $h_{\mathrm{DF}}^{\mathrm{r}}$ is the radial part of the Dirac-Fock operator.

\section{5. $\operatorname{conf}$}

The program conf is based on the code described in 32]. It forms Hamiltonian matrix and the matrix of the operator $\boldsymbol{J}^{2}$ in the configurational space of Slater determinants. These matrices are written to the files CONF.HIJ and CONF. JJJ. Then the matrix eigenvalue equation

$$
\sum_{J \in P^{\mathrm{CI}}} H_{I J} C_{J}=E C_{I}
$$

is solved. Several lower eigenvectors are saved to the file CONF.XIJ. For these eigenvectors the program calculates expectation values $g=\left\langle\boldsymbol{J}^{2}\right\rangle$ and from the quadratic equation $J(J+1)=g$ finds the quantum number $J$ as $J=\sqrt{g+1 / 4}-1 / 2$.

Eigenvalues of the equation (20) include the energy of the core: $E_{i}=E^{\text {core }}+E_{i}^{\text {val }}$. The final table is written to the file CONF.RES and shown on the screen. It presents the quantum numbers $J_{i}$, both $E_{i}$ and $E_{i}^{\text {val }}$ in a.u. and the intervals between energy levels $\Delta_{i}=E_{i}^{\text {val }}-E_{1}^{\text {val }}\left(\right.$ in $\left.\mathrm{cm}^{-1}\right)$, where $E_{1}^{\text {val }}$ is the energy of the lowest-lying level. Note that the signs of all energies are changed to positive. The weights of all configurations contributing to each term are listed in the file CONF. RES under the final table. The quantum numbers $J_{i}$ and eigenvalues $E_{i}$ are also written to the file CONF.XIJ.

An identification of the energy level is based on the knowledge of its total angular momentum $J$ and the weights of the leading configurations. When it is not sufficient, we calculate $g$-factor of the states. This calculation is done by the dtm program, described below.

If the dimension of the matrix Nd in Eq. (20) does not exceed 25000 - 30000, the direct diagonalization of the energy matrix is possible. Direct diagonalization for larger dimensions becomes very time-consuming and other methods should be applied. Typically, we need to find a few low-lying eigenvectors and eigenvalues 
of the Hamiltonian. A method for iterative calculation of a few lowest eigenvalues and eigenvectors of a large real symmetric matrix was suggested by Davidson in Ref. [33]. In this case we diagonalize a block of a reasonable dimension and use respective eigenvectors as a starting approximation for Davidson iterative procedure. This method is applicable to the dimensions of the CI space up to $10^{7}$ and more.

Now we can discuss the structure of the file CONF. INP formed for odd-parity states of Mg I. Let us consider the fragment of the file CONF. INP, generated by the program add. The entire file includes 4340 configurations, but we show only first 6 configurations here as an example.

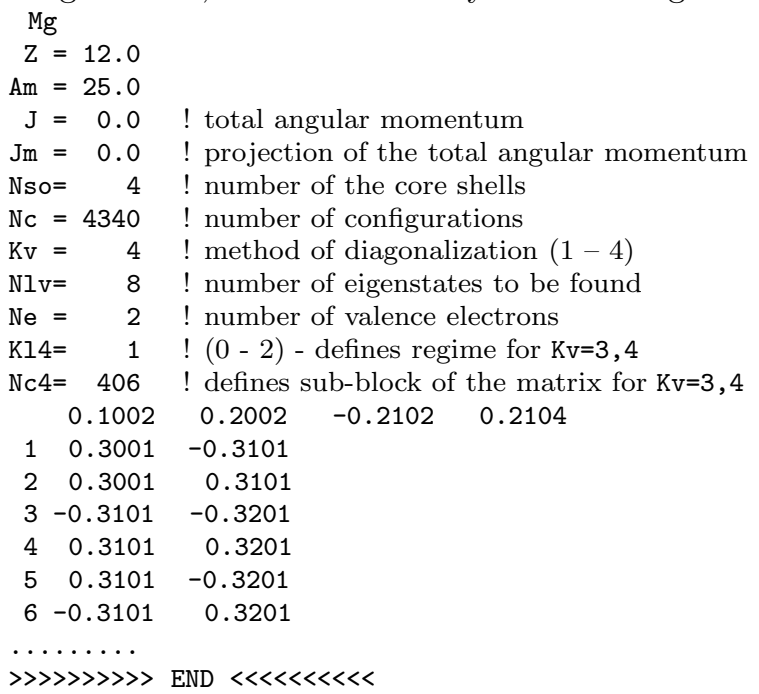

The first three lines are the same as in BASS. INP. Parameter $J$ defines the total angular momentum $J$ of the states we want to calculate. This parameter works only for the keys $\mathrm{Kv}=1,3$ and is ignored for $\mathrm{Kv}=2,4$. Parameter Jm defines projection of the total angular momentum $J_{z}$ for the Slater determinants. Thus, for a given $J_{z}$ we can get only levels with $J \geq J_{z}$. Parameters Nso and Nc define the number of the core shells and total number of configurations.

Parameter Kv specifies a method of diagonalization of the Hamiltonian matrix:

1. $\mathrm{Kv}=1$ - direct diagonalization of the matrix and $J$ selection (only the terms with $J=\mathrm{J}$ will be selected and saved).

2. $\mathrm{Kv}=2$ - direct diagonalization, no $J$ selection.

$3 . \mathrm{Kv}=3$ - Davidson method to find Nlv lower eigenvectors with $J$ selection.

4. $\mathrm{Kv}=4$ - Davidson method without $J$ selection.

The parameters Nlv and Ne define the number of energy levels (eigenvectors) to be found and the number of valence electrons. The key K14 is used only for the Davidson diagonalization, i.e. for Kv=3,4. Standard variant corresponds to $\mathrm{K} 14=1$. In this case we form initial approximation for eigenvectors by diagonalizing the block of the energy matrix. Then, the Davidson procedure is applied. If $\mathrm{K} 14=2$ we use vectors from the file CONF.XIJ as a starting approximation. This option can be chosen if the Davidson procedure did not converge for a specified number of iterations $N_{-} i t$ ( $N \_i t=20$ by default) and more iterations are necessary. Finally, if K14=0 we also use vectors from the file, but make only one iteration.

Parameter Nc4 is also used only for Kv > 2. It specifies the number of configurations, which form the block for the direct diagonalization in the Davidson regime. For larger Nc4 we get better initial approximation and faster convergence, but the time for diagonalization of the block is growing very fast. It may be more important to put leading configurations in the beginning of the list, rather than increasing Nc4 too much.

The optional key Kbrt $=1$ should be added in the head of CONF. INP below the line "Nc4 = ", if we need to include magnetic part of the Breit interaction. By default Kbrt $=0$.

To illustrate how the keys described above work, let us consider two examples:

1. $\mathrm{J}=1, \mathrm{Jm}=1, \mathrm{Kv}=3$, and $\mathrm{Nlv}=10$ - the Davidson method of diagonalization will be applied and 10 lowest-lying states with $J=1$ will be found. 
2. $\mathrm{Jm}=2, \mathrm{Kv}=2$, and $\mathrm{Nlv}=20$ - the Hamiltonian will be directly diagonalized and 20 lowest states with $J \geq 2$ will be found.

Next part of the file CONF. INP contains the list of core shells followed by the list of configurations. The format of presentation of the quantum numbers and occupations numbers of the shells as a single real number is the same as in the files BASS. INP and ADD. INP. The configuration list may include empty lines. Each configuration should contain Ne electrons. In our example six relativistic configurations, which are shown explicitly, belong to two nonrelativistic configurations $3 s 3 p$ and $3 p 3 d$. The number of core shells must be equal to Nso. If the actual number of configurations exceeds Nc, all extra configurations will be ignored.

In addition to the input from the file there is a dialog in the beginning of execution of the conf program. It starts with the message

$\mathrm{KI}=$ (0-Start, 1-Cont. , 2-MBPT , 3-Add):

and we have 4 options to choose from:

1. $\mathrm{KI}=0$ - pure $\mathrm{CI}$ calculation from the beginning. We start with construction of the energy matrix, then it is diagonalized.

2. $\mathrm{KI}=1-$ continuation of the previous calculation. The program checks for the files and starts where it stopped before. If the file CONF.HIJ is complete, the matrix is not recalculated. If the file exists, but the matrix calculation was not completed, the program tries to continue construction of the matrix and then starts diagonalization. Typically this key is used to repeat the Davidson iteration procedure until the convergence is reached (requires the key $\mathrm{K} 14=2$ in the file CONF. INP).

3. $\mathrm{KI}=2-\mathrm{CI}+\mathrm{MBPT}$ calculation from the beginning. The matrix is formed using the files SGC.CON and SCRC. CON in addition to the file CONF. INT. In this case there is additional dialog, which we discuss below.

4. $\mathrm{KI}=3-$ extending previous calculation, when new configurations were added to the end of the list in the file CONF. INP. The matrix is extended without recalculating already existing part. If the key $\mathrm{K} 14=2$ is used, then eigenvectors from the previous calculation are used as a starting approximation for the Davidson procedure. This key is useful for very large calculations. It may also allow us to avoid using large blocks Nc4 for Davidson procedure.

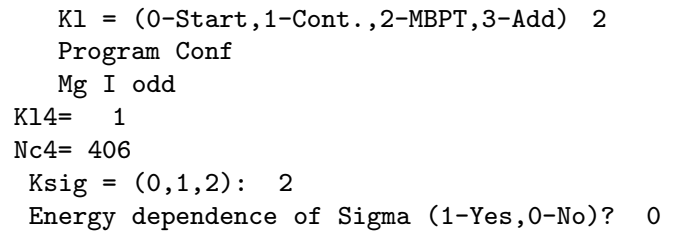

Figure 3: An example of the dialog with program conf.

There is an additional dialog for the CI+MBPT calculation, i.e., for $\mathrm{Kl}=2$ (see Fig. 31).

Message: $\quad \mathrm{Ksig}=(0,1,2)$ :

$\overline{\text { Choosing }} 0$ we return to the pure CI calculation (i.e. the same as the variant Kl $=0$ ). If we choose Ksig $=1$, then only one-electron (self-energy) MBPT corrections are included. For Ksig $=2$ both one- and two-electron (self-energy and screening) MBPT corrections are included.

Message: Energy dependence of Sigma (1-Yes,0-No)?

- 0 (recommended) - automatic approximation of the energy-dependence of the operator $\Sigma(E)$.

- 1 - the energy $E$ is introduced manually to treat energy-dependence more accurately.

If we choose ' 1 ' (the energy-dependence), then we get

Message: Give valence energy:

We need to specify the energy $E_{\mathrm{val}}$, for which the effective Hamiltonian (8) will be formed. Note that here the energy of the bound state should be given as a negative number (note that in the output to the screen and to the file CONF.RES the signs for all energies are changed to positive). 
The dtm program calculates matrix elements of one-electron operators between many-electron states. We use the formalism of the density, or transition matrix and the name of the program comes from the resulting abbreviation. At present the total number of one-electron operators included in dtm is 13 . The following quantities can be calculated: electronic $g$-factors; magnetic dipole and electric quadrupole hyperfine structure constants $A$ and $B$; the amplitudes of electric $(E k)$ and magnetic $(M k)$ multipole transitions, where $k=1,2,3$ correspond to the dipole, quadrupole, and octupole transitions; the nuclear spin independent parity nonconserving (PNC) amplitude and the amplitude of the electron interaction with the P-odd nuclear anapole moment $(\mathrm{AM}) \sqrt{2}$; the $\mathrm{P}, \mathrm{T}$-odd interaction of the electron electric dipole moment and the nucleus magnetic quadrupole moment.

The formalism of transition matrix allows us to express the matrix elements between many-electron states via one-electron matrix elements. Let us consider the matrix element $\left\langle J^{\prime} M^{\prime}\left|T_{q}^{L}\right| J M\right\rangle$, where $|J M\rangle$ and $\left|J^{\prime} M^{\prime}\right\rangle$ are the many-electron states with the total angular momenta $J^{\prime}, J$ and their projections $M^{\prime}, M$, and $T_{q}^{L}$ is the spherical component of the tensor operator $T$ of rank $L$. Transition matrix is defined a 3 :

$$
\begin{aligned}
\hat{\rho} & \equiv \rho_{n l j m, n^{\prime} l^{\prime} j^{\prime} m^{\prime}}|n l j m\rangle\left\langle n^{\prime} l^{\prime} j^{\prime} m^{\prime}\right|, \\
\rho_{n l j m, n^{\prime} l^{\prime} j^{\prime} m^{\prime}} & =\left\langle J^{\prime} M^{\prime}\left|a_{n^{\prime} l^{\prime} j^{\prime} m^{\prime}}^{\dagger} a_{n l j m}\right| J M\right\rangle
\end{aligned}
$$

where $|n l j m\rangle$ is the one-electron state. Then the many-electron matrix element is reduced to

$$
\left\langle J^{\prime} M^{\prime}\left|T_{q}^{L}\right| J M\right\rangle=\operatorname{Tr} \rho_{n l j m, n^{\prime} l^{\prime} j^{\prime} m^{\prime}}\left\langle n^{\prime} l^{\prime} j^{\prime} m^{\prime}\left|T_{q}^{L}\right| n l j m\right\rangle,
$$

where "Tr" (trace) means the summation over all quantum numbers $n, l, j, m ; n^{\prime}, l^{\prime}, j^{\prime}, m^{\prime}$.

Using the Wigner-Eckart theorem we can obtain expression similar to (23) for the reduced matrix elements. If we determine the reduced transition matrix of the rank $L$ as

$$
\rho_{n l j, n^{\prime} l^{\prime} j^{\prime}}^{L}=(-1)^{J^{\prime}-M^{\prime}}\left(\begin{array}{rcc}
J^{\prime} & L & J \\
-M^{\prime} & q & M
\end{array}\right)^{-1} \sum_{m m^{\prime}}(-1)^{j^{\prime}-m^{\prime}}\left(\begin{array}{rcc}
j^{\prime} & L & j \\
-m^{\prime} & q & m
\end{array}\right) \rho_{n l j m, n^{\prime} l^{\prime} j^{\prime} m^{\prime}}^{L},
$$

we arrive at

$$
\left\langle J^{\prime}\left\|T^{L}\right\| J\right\rangle=\operatorname{Tr} \rho_{n l j, n^{\prime} l^{\prime} j^{\prime}}^{L}\left\langle n^{\prime} l^{\prime} j^{\prime}\left\|T^{L}\right\| n l j\right\rangle .
$$

The dtm program forms reduced density (or transition) matrices of ranks $L=0-3$ and calculates the reduced matrix elements (25). It uses the same input file CONF. INP as the program conf. This file was described in Section 4.5. It can include two optional parameters Gnuc and Qnuc that are used for calculating the magnetic dipole and electric quadrupole hyperfine structure constants $A$ and $B$. They can be added to the head of the CONF. INP after the line Nc4=... Parameter Gnuc gives the gyromagnetic ratio $\frac{1}{I} \frac{\mu_{I}}{\mu_{N}}$, where $I$ is the nuclear spin and $\mu_{I}$ is the nuclear magnetic moment expressed in nuclear magnetons $\mu_{N}$. Parameter Qnuc is the nuclear quadrupole moment expressed in barns. By default Gnuc $=1$ and Qnuc $=1$.

We need density matrix to calculate $g$-factors and hyperfine structure constants. It follows from Eq. (24) that for $M^{\prime}=M=0$ the respective $3 j$-symbol turns to zero if $\left(J^{\prime}+L+J\right)$ is odd. To avoid such a problem we should use $\mathrm{Jm} \neq 0$, at least, for one of the states $|J M\rangle$ or $\left|J^{\prime} M^{\prime}\right\rangle$. For the diagonal matrix elements the dtm program needs binary files CONF. DAT, CONF. DET and CONF.XIJ. The program reads the list of determinants from CONF.DET and the eigenvectors from CONF. XIJ.

If we want to find a non-diagonal matrix element (for instance, an E1 transition amplitude), we need lists of the determinants and the eigenvectors for both states. The initial state is defined by the files CONF.DET and CONF.XIJ and final states are defined by the files CONF1.DET and CONF1.XIJ. The results

\footnotetext{
${ }^{1}$ The $E 1$ transition amplitudes are calculated in the length $(\mathrm{L})$ and velocity $(\mathrm{V})$ gauges.

${ }^{2}$ Note that interaction with the nuclear AM is indistinguishable from the nuclear spin dependent PNC interaction.

${ }^{3}$ The density matrix corresponds to the special case $\left|J^{\prime}, M^{\prime}\right\rangle=|J, M\rangle$.
} 

TM. RES, correspondingly.

When we run the dtm program we should interactively choose between two options:

\section{Message: Do you want DM (1) OR TM (2)?}

1. If the answer is "1", density matrix (DM) and diagonal matrix elements are calculated.

- Message: DM for terms .. - . : :

Two numbers should be specified. These are the serial numbers of the energy levels listed in the output file CONF.RES. The eigenvectors in the file CONF.XIJ are written in the same order. Example:

Do you want DM (1) OR TM (2)?

1

DM for terms .. - . :

24

If the numbers 2 and 4 are entered, the program calculates three diagonal matrix elements $(\langle i|\ldots| i\rangle, i=2,3,4)$ for all operators which are allowed by the selection rules.

2. If the answer is "2", transition matrix (TM) and non-diagonal matrix elements are calculated.

- Message: TM from term .. to terms . . - . :

Three numbers should be specified. First one is the serial number of the initial state. This eigenvector will be read from CONF.XIJ. Two other numbers are the serial numbers of the final states taken from CONF1.XIJ.

Example:

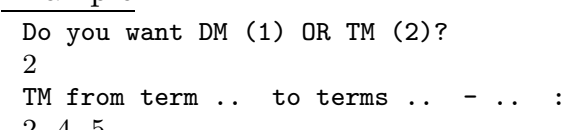

245

If we enter numbers $2,4,5$, the program calculates two non-diagonal matrix elements $(\langle f|\ldots| 2\rangle, f=$ $4,5)$ for all operators, which are allowed by the selection rules.

The dtm program creates the file DTM. INT with radial integrals for all 13 operators and the text file DM.RES or TM.RES with the results. If the DTM. INT file already exists, dtm uses it and does not recalculate radial integrals. This is important for including random-phase approximation (RPA) corrections. We discuss RPA corrections in more detail in Section 5.3 .

\section{Description of programs to include MBPT}

In this section we describe the programs that calculate corrections to the radial integrals for valence electrons, which account for the core-valence correlations. In Section 2 we introduced operator $\Sigma$, Eq. (9), which is added to the valence Hamiltonian. A detailed description of the MBPT approach, including analytical expressions and Brueckner-Goldston diagrams can be found in [16].

By analogy with the effective Hamiltonian, Eq. (8), we introduce effective one-electron operators $\left(T_{q}^{L}\right)_{\text {eff }}$ for the quantities calculated by $\mathrm{dtm}$. These operators act in the valence space $P$. We determine them using the random-phase approximation (RPA) [26, 34, 35]. Qualitatively, the RPA describes a shielding of the externally applied field by the core electrons. It is essential to include these corrections in any high-accuracy calculations. Sometimes they change "bare" matrix elements by $30-40 \%$.

In order to construct effective Hamiltonian we need to calculate effective one- and two-electron matrix elements. The former are described by a set of one-electron (self-energy) diagrams, which are calculated by the sgc program. Two-electron amplitudes correspond to the screening diagrams. It is important that angular parts for these diagrams are the same as for the Coulomb matrix elements. This allows us to present them in the form of the effective radial integrals, which are calculated by the scrc program. Third program, rpa, calculates radial integrals of the effective operators within RPA approximation. Below we describe these programs one by one. 
All three programs of the MBPT block of the package use the same input file MBPT. INP and binary files with the orbitals HFD.DAT and CONF.DAT. The input file consists of several independent parts and different programs read different parts of the file. Each part is marked by the label and its position in the file is arbitrary. We will discuss these parts of the input separately. An example of the whole file for the calculation of $\mathrm{Mg} \mathrm{I}$ is given in Fig. 4

\section{1. $\mathrm{sgc}$}

The sgc program calculates one-electron effective radial integrals of the operator $\Sigma$, Eq. (9), and writes them to the ASCII file SGC.CON. It reads the parts of the input file marked with labels MBPT, SBT, Sig, and Eval. Below we describe each of these blocks.

The block MBPT is used by all three programs and includes the most general parameters and keys.

1. Nso is the number of core shells not included in CI (the same as in CONF. INP).

2. Nsh is the number of shells which form the Hartree-Fock potential. These are the shells for which NC $=0$ in HFD. INP.

3. Nss defines last virtual orbital for internal summations in diagrams. If Nss $\geq N \mathbf{N}$, where Ns is the total number of orbitals in the basis set, then the sums run over all Ns orbitals.

4. Nsv is the first valence orbital for which radial integrals will be calculated. Usually Nsv $=$ Nso +1 .

5. Nmax: for orbitals above Nmax all effective radial integrals are neglected.

6. Lmax: for orbitals with $l>$ Lmax all effective radial integrals are neglected.

7. Kmax: Coulomb matrix elements with multipolarity $k>$ Kmax are neglected.

8. $\mathrm{Kt}=0,1$ determines the step $h$ for numerical integration, $h=2-\mathrm{Kt}$. Using $\mathrm{Kt}=0$ speeds up integration at the expense of the accuracy.

9. Kbrt is read only by rpa, but is ignored by sgc and scrc.

10. Kout defines the level of details in the output files. Kout $=0$ corresponds to the shortest output and Kout $=2$ corresponds to most detailed output.

The block SBT is read only if Nsh > Nso. When some of the valence electrons contribute to the HF field we get an additional class of the so called "subtraction" diagrams [16]. For each of Nso+1, ... Nsv orbitals we need to define occupation numbers, which were used to produce the HF field. In Fig. 4 we have Nso=Nsh=4 and the block SBT is absent. If both $3 s$ electrons are included in the initial HFD self-consistency procedure (it corresponds to $V^{N}$ approximation), Nsh $=5$ and this block looks as:

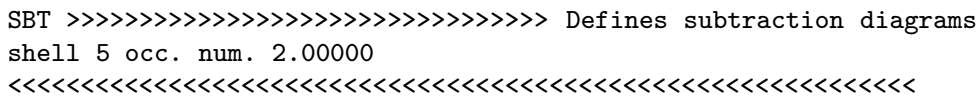

In this case subtraction diagrams account for the field of two valence electrons on the 5 th shell, i.e., the field of $3 s^{2}$ electrons.

The block Sig includes several additional parameters and keys for the programs sgc and scrc.

1. Khot $=0,1$. If Khot $=1$, then high-order corrections to the second order MBPT are approximated with the help of the "screening coefficients" (see below).

2. Parameters Nav, Nsx1, Nsx2 are used to speed up calculation of diagrams without significant loss of accuracy. When we calculate diagrams for the matrix element $\left\langle n_{a}\left|\Sigma^{(1)}\right| n_{b}\right\rangle$, where $n_{a}$ and $n_{b}$ numerate orbitals in the basis set, we sum over excitations from the core shells. The number of upper core shells involved in summations is given by the parameters Nsx1 and Nsx2. If $\left(n_{a}+n_{b}\right) / 2 \leq$ Nav, the sums run over Nsx1 core shells, otherwise over Nsx2.

Typically we put Nsx1=Nso, Nsx2=0. In this case, if $\mathrm{Nav}=$ Nmax, all matrix elements are calculated. This is the standard choice because calculations of self-energy corrections are not very time-consuming. If Nav < Nmax, the matrix elements for $\left(n_{a}+n_{b}\right) / 2>$ Nav are not calculated. 
MBPT>>>>>>>>>>>>>>>>>>>>>>>>>>>>>>>>>>>>Used by ALL MBPT programs

Nso $=4$

$\mathrm{Nsh}=4$

Nss $=999$ - last virtual orbital to include in RPA

$\mathrm{Nsv}=5$

Nmax $=151$

$\operatorname{Lmax}=3$

$\mathrm{Kmax}=7$

$\mathrm{Kt}=1-$ in radial integrals step $=2-\mathrm{Kt}$

$\mathrm{Kbrt}=0$ - Breit interaction is not included yet

Kout $=0$ - Details in output file

Kan
R

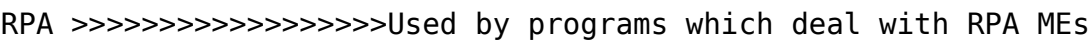

A hf 1 |

B-hf 0

Ề L 1

EDM $\quad 0 \quad$ - Right hand side operators

PNC 0

E1_V 1 | put ' 1 ' for all operators you are

$\mathrm{AM}^{-} \quad 0 \quad$ interested in

MQM $\quad 0$

M1 0

E2 0

E3 0

M2 0

M3 0

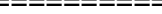

Nhf $=4$

$\mathrm{Kmg}=0$ - if not zero, Omega gives frequency of external field Omega $=0.0000 \quad$ (a.u.)

Kex $=1$ - (if Kex=0 then exchange RPA diagrams are skipped)

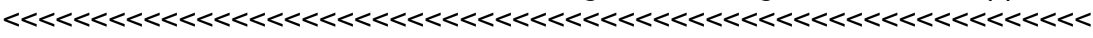
Sig: Khot $=0$ - if Khot $=1$ then high order terms are included

$\mathrm{Nav}=82$

$\mathrm{Ns} \times 1=4$

Ns $\times 2=0$ - number of active core shells

$\mathrm{K}$ inf 0 - extrapolation to $\mathrm{L} \max =$ infinity $[0,1,3,5]$

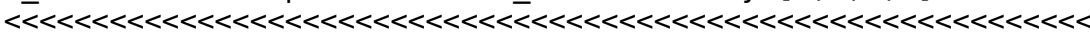

Eval $>>>>>>>>>>>>>>>>>>>>>>>>>>>>>>>>>$ Used by ALL MBPT programs

$\mathrm{Kval}=1$ - defines valence energies: $0-\mathrm{HF} ; 1$-min(HF); 2-given below

$0 \quad-0.54140$

$\begin{array}{lll}1 & -0.38407 & -0.38366\end{array}$

$2-0.22481-0.22482$

$3-0.00000-0.00000$

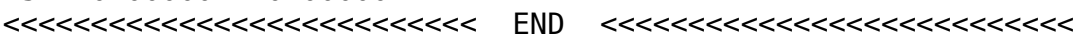

Figure 4: Example of the input file MBPT. INP to calculate MBPT corrections for atomic Mg 
3. The key K_inf $=0,1,3,5$ switches on/off extrapolation to the $L_{\max } \rightarrow \infty$, where $L_{\max }+1$ is the number of the partial waves $\left(s, p, d\right.$, etc.) in the basis set. For $\mathrm{K}_{-}$inf $=0$ there is no extrapolation. For K_inf $=1,3,5$ the program approximates contribution of the missing partial waves with the function $f_{a}(l)=A /(l+a)^{3}$, where $a=0,-0.5,-1$ respectively.

The block Eval is used to define energy denominators in the diagrams. For the intermediate states we always use Dirac-Fock energies, but for the initial orbitals we have three variants. Standard variant corresponds to $\mathrm{Kval}=1$.

1. Kval $=0$ - for all initial orbitals we use their Dirac-Fock energies.

2. Kval $=1-$ for all orbitals of a given partial wave we use the lowest valence energy in this wave.

3. Kval $=2$ - for all orbitals of a given partial wave we use the energy from the list in the file MBPT. INP. This list includes quantum numbers $l$ followed by one, or two energies for the respective relativistic partial wave(s) (see example in Fig. (4).

The block HOT (high-order terms) is read only if Khot $=1$. In this case, when the diagrams are calculated, the Coulomb radial integrals of the multipolarity $k$ are multiplied by the screening coefficient Chot $k$. These coefficients account for the screening of the interaction between electrons by the polarization of the core. For example, we can have:

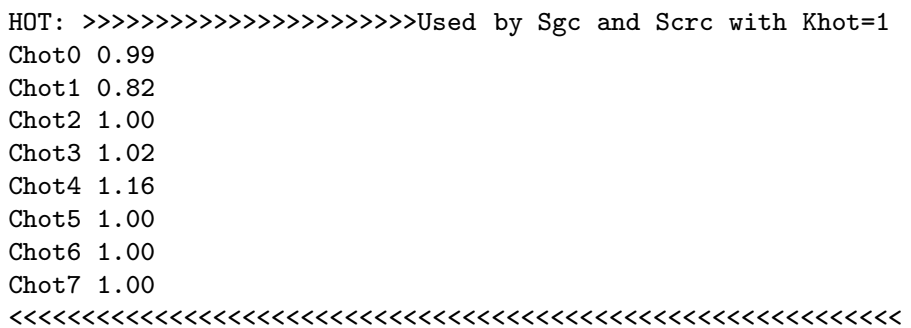

\section{2. $\mathrm{scrc}$}

The scrc program needs the same blocks of the input file as the sgc program. Here the meaning of the parameter Nav is slightly different. Now we calculate two-electron matrix elements $\left\langle n_{a} n_{b}\left|\Sigma^{(2)}\right| n_{c} n_{d}\right\rangle$. If $\left(n_{a}+n_{b}+n_{c}+n_{d}\right) / 4 \leq \mathrm{Nav}$, the sums run over Nsx1 core shells, otherwise over Nsx2. The total number of the two-electron diagrams may be very large and calculations are much more time-consuming then for the one-electron diagrams. Typically the MBPT corrections for the orbitals with large principle quantum numbers are very small and respective two-electron effective radial integrals can be neglected. If Nsx1=Nso, Nsx2 $=0$ it can be done by choosing Nav < Nmax.

Let us illustrate a possible choice of Nav by the following example. Suppose, we are interested in calculation of an energy level of $\mathrm{Mg} \mathrm{I}$, whose main configuration is $\left(3 s 5 d_{5 / 2}\right)$. Let the serial number of the $5 d_{5 / 2}$ orbital in the basis set be equal 21 , while the total number of orbitals in the basis set is 120 . Then we can determine Nav as $(3 \times 21+120) / 4 \approx 46$. In this way we accurately account for the MBPT effects for all singly excited configurations in the CI space, but not for all double excitations. Usually this approximation is adequate.

The interpolation key K_inf is not used for the two-electron diagrams. The output files are the binary file SCRC.CON and the text file with results SCRC.RES.

\section{3. $\mathrm{rpa}$}

The rpa program solves RPA equations, calculates radial integrals of the effective (dressed) operators and writes them to the files RPA_n. INT. Here the number $n=1-13$ numerates one-electron operators in the same order as they are listed in the block RPA of the file MBPT. INP. For example, for the PNC operator, the file RPA_5.INT will be created, etc. The output is also written to the text file RPA.RES.

The rpa program reads three blocks of the input file, namely MBPT, Eval, and RPA. Two of them were described in Section 5.1 The block RPA starts with the list of all 13 operators, which are currently coded: 
1. A_hf - magnetic dipole hyperfine constant.

2. B_hf - electric quadrupole hyperfine constant.

3. E1_L - electric dipole transition amplitude in the length gauge.

4. EDM - Parity (P)-odd, Time (T)-odd interaction of the electric dipole moment of the electron.

5. PNC - P-odd nuclear spin-independent parity-nonconserving interaction.

6. E1_V - electric dipole transition amplitude in the velocity gauge.

7. AM - P-odd nuclear spin-dependent parity-nonconserving interaction, or the interaction with the P-odd nuclear anapole moment.

8. MQM - P,T-odd interaction with the magnetic quadrupole moment of the nucleus.

9. M1 - magnetic dipole transition amplitude.

10. E2 - electric quadrupole transition amplitude.

11. E3 - electric octupole transition amplitude.

12. M2 - magnetic quadrupole transition amplitude.

13. M3 - magnetic octupole transition amplitude.

The numbers 0 or 1 after the operator tells the program to skip this operator or carry out calculations for it. The meaning of the remaining parameters is the following.

- Nhf - the SCF procedure includes Nhf upper orbitals (Nsh, Nsh-1, ...). This parameter is similar to Nsx1 and Nsx2 used by sgc. Recommended value is Nhf=Nsh.

- $\mathrm{Kmg}=0,1$ - if $\mathrm{Kmg}=1$, the frequency of the external field will be read on the next line.

- Omega - the frequency of the external field (in a.u.). For $\mathrm{Kmg}=0$ the frequency is put equal 0 . This corresponds to the static limit of the RPA equations. Usually static approximation is adequate even for the electromagnetic transitions, which correspond to the optical frequencies.

- $\mathrm{Kex}=0,1$ - for $K e x=0$ the exchange term in the RPA equations is neglected. Standard variant corresponds to $\mathrm{Kex}=1$.

At the beginning of the execution we need to choose between two options,

RPA for core (1) or for valence (2):

If we choose (1), the RPA equations are solved only for the core orbitals. If we choose (2) the program may skip solution for the core if the files RPA_n. INT are present and calculate RPA integrals for the valence orbitals. If the files are absent, or do not match parameters of the basis set, the program starts with the RPA equations for the core and then calculates valence integrals.

\section{4. rpa_dtm}

The rpa_dtm program rewrites the radial integrals of "dressed" operators from the RPA*. INT files to the DTM. INT file. Subsequently, this DTM. INT file can be used by the dtm program for calculating matrix elements. In such a way we take into account the RPA corrections and find the matrix elements of the "dressed" operators. Note that before running the program rpa_dtm we need to form the file DTM. INT with the help of the program dtm. If not all RPA*. INT files are present, the program keeps "bare" radial integrals for the respective operators.

\section{Calculation of $\mathrm{Mg}$}

In this Section we give an example how the entire code package works. The corresponding sets of input and output files are given in directories "INPUT" and "OUTPUT". All script files (*.sh), illustrating how to perform $\mathrm{Mg}$ calculations, are also included in the package. We choose Mg as a two-valence system with a small core where calculations are not very time consuming, but still give full example of the package capabilities. We already used $\mathrm{Mg}$ as an example when discussing inputs for different programs. Present calculations are carried out with the same input files. 


\subsection{Basis set}

The basis set for the calculation was formed as described above in Sections 4.1 and 4.2 We started from solution of relativistic Hartree-Fock equations for the core $\left[1 s^{2} 2 s^{2} 2 p^{6}\right]$ electrons and obtained $1 s, 2 s$, and $2 p$ orbitals. Then the core orbitals were frozen and the Hartree-Fock $3 s, 4 s, 3 p, 4 p, 3 d, 4 d$, and $4 f$ orbitals were constructed in the frozen-core potential. Other (virtual) orbitals were constructed in the regime "auto" as described in Section 4.2. The basis set included the $s, p, d, f, g$, and $h$ orbitals with principal quantum number $n \leq 25$. In the following we use designation [25spdf $g h]$.

The CI space (set of configurations) was formed by single (S) and double (D) excitations from the nonrelativistic $3 s^{2}, 3 s 4 s$, and $3 s 3 d$ configurations (for even-parity states) and from $3 s 3 p$ configurations (for odd-parity states). Both single and double excitations are allowed to $15 \operatorname{spdfg}$ orbitals ([15spdfg (S+D)]), and only single excitations are allowed to 16-18 spdf orbitals ([18spdf (S)]).

Further increase of the CI space to [18spd15fg (S+D)] and [20spdfg (S)] led to change of energies at the level of $0.01-0.02 \%$. The matrix elements of the allowed $E 1$ transitions changed at most by $0.06 \%$. Therefore, the basis set is numerically complete and the CI space is sufficiently saturated in our example.

\subsection{Energy levels}

In Table 2 we present calculated energies of the low-lying states of $\mathrm{Mg}$. Calculations were done in the framework of the conventional two-electron CI as well as using the formalism of CI+MBPT approach. For the CI method the agreement between calculated and experimental energies is at the level of 1.7-4.5\%. The combination of CI and MBPT improves the accuracy by approximately an order of magnitude. Our results are compared with the experimental data and energy levels obtained by Cheng et al. 36] in the frame of the multi-configuration Dirac-Fock self-consistent field (MCDF-SCF) and relativistic configuration interaction (RCI) methods, using the GRASP-2K program [7] with a quasi-complete basis set (the principal quantum number of the pseudo-orbitals $\tilde{n} \leq 8$ and the included partial waves were $s, p, d, f, g, h, i, k)$. We did not include the Breit corrections. For consistency we present results of Ref. [36] also without Breit corrections. It is shown in [36] that these corrections do not exceed $3 \mathrm{~cm}^{-1}$ for all presented levels.

Table 2: The two-electron binding energies $\left(\right.$ in $\mathrm{cm}^{-1}$ ) are given in the first row for the ground state $\left(1 \mathrm{a} \cdot \mathrm{u} . \approx 219474.63 \mathrm{~cm}^{-1}\right)$. In other rows the energy differences $\Delta$ are counted from the ground state. The differences with the experimental values are given in percent for all calculations. In columns 3-6 we present our results obtained using the CI and CI+MBPT methods. In columns 7 and 8 the results of Cheng et al. [36] obtained using the MCDF-SCF method with the GRASP-2K program are given.

\begin{tabular}{ccccccccc}
\hline & & \multicolumn{3}{c}{ CI } & \multicolumn{2}{c}{ CI+MBPT } & MCDF-SCF [36] & Experiment [37] \\
Config. & Level & $\Delta$ & $\%$ & $\Delta$ & $\%$ & $\Delta$ & $\%$ & $\Delta$ \\
\hline $3 s^{2}$ & ${ }^{1} S_{0}$ & 179534 & -1.9 & 182645 & -0.2 & - & - & $182939^{\mathrm{a}}$ \\
$3 s 4 s$ & ${ }^{3} S_{1}$ & 40397 & -2.0 & 41107 & -0.2 & 41368 & 0.4 & 41197 \\
$3 s 4 s$ & ${ }^{1} S_{0}$ & 42663 & -2.0 & 43418 & -0.2 & 43690 & 0.4 & 43503 \\
$3 s 3 d$ & ${ }^{1} D_{2}$ & 45105 & -2.9 & 46281 & -0.3 & 46619 & 0.5 & 46403 \\
$3 s 3 d$ & ${ }^{3} D_{1}$ & 46951.11 & -2.1 & 47849.75 & -0.2 & 48169.2 & 0.4 & 47957.06 \\
$3 s 3 d$ & ${ }^{3} D_{2}$ & 46951.16 & -2.1 & 47849.79 & -0.2 & 48169.3 & 0.4 & 47957.03 \\
$3 s 3 d$ & ${ }^{3} D_{3}$ & 46951.24 & -2.1 & 47849.85 & -0.2 & 48169.3 & 0.4 & 47957.05 \\
$3 s 3 p$ & ${ }^{3} P_{0}^{o}$ & 20902 & -4.5 & 21765 & -0.4 & 21870 & 0.1 & 21850 \\
$3 s 3 p$ & ${ }^{3} P_{1}^{o}$ & 20923 & -4.5 & 21786 & -0.4 & 21892 & 0.1 & 21870 \\
$3 s 3 p$ & ${ }^{3} P_{2}^{o}$ & 20963 & -4.5 & 21829 & -0.4 & 21935 & 0.1 & 21911 \\
$3 s 3 p$ & ${ }^{1} P_{1}^{o}$ & 34467 & -1.7 & 35018 & -0.1 & 35184 & 0.4 & 35051 \\
$3 s 4 p$ & ${ }^{3} P_{0}^{o}$ & 46922 & -2.0 & 47751 & -0.2 & 48011 & 0.4 & 47841 \\
$3 s 4 p$ & ${ }^{3} P_{1}^{o}$ & 46926 & -2.0 & 47754 & -0.2 & 48015 & 0.4 & 47844 \\
$3 s 4 p$ & ${ }^{3} P_{2}^{o}$ & 46933 & -2.0 & 47761 & -0.2 & 48022 & 0.4 & 47851 \\
$3 s 4 p$ & ${ }^{1} P_{1}^{o}$ & 48515 & -1.7 & 49294 & -0.1 & 49532 & 0.4 & 49347 \\
\hline
\end{tabular}

${ }^{a}$ The two-electron binding energy of the ground state is equal to the sum of the first two ionization potentials $\operatorname{IP}(\mathrm{Mg})+\operatorname{IP}\left(\mathrm{Mg}^{+}\right)$, where $\operatorname{IP}(\mathrm{Mg})=61671.1 \mathrm{~cm}^{-1}$ and $\operatorname{IP}\left(\mathrm{Mg}^{+}\right)=121267.6 \mathrm{~cm}^{-1}$ 37]. 
Table 3: Absolute values of the reduced $E 1$ matrix elements (in atomic units) for the $3 s^{2}{ }^{1} S_{0} \rightarrow 3 s 3 p^{3,1} P_{1}^{o}$ and $3 s 4 s{ }^{1} S_{0} \rightarrow$ $3 s 3 p^{3,1} P_{1}^{o}$ transitions in $\mathrm{Mg}$. Our CI and CI+MBPT results are compared with other available data. Brief description of other theoretical methods is given in the text. The results obtained in the length and velocity gauges are presented in columns labeled " $L$ " and " $V$ "

\begin{tabular}{|c|c|c|c|c|c|c|c|c|}
\hline \multirow[b]{2}{*}{ Method } & \multicolumn{2}{|c|}{$\left\langle 3 s 3 p^{3} P_{1}^{o}\|D\| 3 s^{2}{ }^{1} S_{0}\right\rangle$} & \multicolumn{2}{|c|}{$\left\langle 3 s 3 p^{1} P_{1}^{o}\|D\| 3 s^{2}{ }^{1} S_{0}\right\rangle$} & \multicolumn{2}{|c|}{$\left\langle 3 s 3 p^{3} P_{1}^{o}\|D\| 3 s 4 s^{1} S_{0}\right\rangle$} & \multicolumn{2}{|c|}{$\left\langle 3 s 3 p^{1} P_{1}^{o}\|D\| 3 s 4 s^{1} S_{0}\right\rangle$} \\
\hline & $L$ & V & $L$ & $V$ & $L$ & V & $L$ & $V$ \\
\hline CI & 0.00548 & 0.00634 & 4.10 & 4.07 & 0.00295 & 0.00317 & 4.33 & 4.29 \\
\hline $\mathrm{CI}+\mathrm{MBPT}$ & 0.00599 & 0.00646 & 4.03 & 4.06 & 0.00314 & 0.00328 & 4.26 & 4.24 \\
\hline MCDF-SCF ${ }^{a}$ & 0.0059 & & 4.01 & & & & & \\
\hline $\mathrm{MCDF}-\mathrm{SCF}+\mathrm{Br}^{\mathrm{a}}$ & 0.0055 & & 4.01 & & & & & \\
\hline $\mathrm{MCDF}^{\mathrm{b}}$ & & & 3.99 & 4.02 & & & & \\
\hline $\mathrm{MCHF}+\mathrm{BP}^{\mathrm{c}}$ & & & & & 0.00324 & 0.00337 & 4.26 & 4.31 \\
\hline Experiment & \multicolumn{2}{|c|}{$0.0053(3)^{\mathrm{d}}$} & \multicolumn{2}{|c|}{$4.15(10)^{\mathrm{f}}$} & & & & \\
\hline & \multicolumn{2}{|c|}{$0.0056(4)^{\mathrm{e}}$} & \multicolumn{2}{|c|}{$4.06(10)^{\mathrm{g}}$} & & & & \\
\hline
\end{tabular}

${ }^{a}$ Reference [38]. Recalculation from the oscillator strengths.

${ }^{b}$ Reference [39]. Recalculation from the oscillator strengths.

${ }^{\mathrm{c}}$ Reference [40].

${ }^{\mathrm{d}}$ Reference [41].

e Reference [42].

${ }^{\mathrm{f}}$ Reference [43].

gReference [44].

\section{Acknowledgements}

We thank V. A. Dzuba and V. V. Flambaum for many useful discussions and E. Konovalova for a help with calculations. This work was supported in part by U.S. NSF Grant No. PHY-1212442. MGK acknowledges support from RFBR Grant No. 14-02-00241. IIT acknowledges support from RFBR Grant No. 15-03-07644.

\section{Appendix A. Auxiliary files}

Auxiliary files (with extension inc) include subroutines used by one, or more main programs with the help of the operator include. Below we briefly describe these codes. We start with the * inc files used by the specified program(s) and then we list the $*$.inc files used by several programs.

1. bass.for and basc.for: 
(a) bas1.inc - a set of subroutines which deal with the orbitals (form them, orthogonalize them, etc.).

(b) bspl.inc - two subroutines to form $B$ splines, which may be used to construct virtual orbitals.

(c) breit.inc - four subroutines which calculate Breit interaction.

(d) test_ori.inc - tests Taylor expansion of the radial functions at the origin.

2. conf.for:

(a) hread.inc-reads/writes the energy matrix to/from the file CONF.HIJ.

(b) gaunt.inc - finds the angular part of the two-electron interaction.

(c) hint.inc - calculates one- and two-electron matrix elements for the energy matrix.

(d) formj.inc - forms the matrix of the operator $\boldsymbol{J}^{2}$ and writes it to the file CONF. JJJ.

3. sgc.for and scrc.for:

(a) sg1.inc - evaluates one-electron diagrams for the operator $\Sigma$ (used by sgc.for).

(b) scr1.inc - evaluates two-electron diagrams for the operator $\Sigma$ (used by scrc.for).

(c) cfnc.inc - a set of small subroutines used to calculate diagrams.

(d) rp2.inc - calculates radial part of the second order MBPT diagram.

4. *. inc files used by several programs:

(a) hould.inc - direct diagonalization of a symmetric matrix.

(b) sint1.inc - numerical integration on the radial grid.

(c) dif.inc - numerical differentiation of the radial functions.

(d) wig.inc - calculates $3 j-, 6 j$-, and $9 j$-symbols.

(e) readf.inc and readff.inc - read two-component radial functions either directly from the files HFD.DAT and CONF.DAT, or from the arrays ArrP and ArrQ.

(f) inpstr.inc - reads optional parameters in the input files.

(g) pi_pk.inc - calculates radial integrals for the specific mass shift operator.

(h) yk.inc - evaluates the Coulomb function $Y(\kappa, r)$.

(i) dets.inc - a set of subroutines for operations with Slater determinants.

(j) eval.inc - defines valence energies of the orbitals for MBPT calculations.

(k) check_dim.inc - checks consistency of the parameters in the files conf.par and rpa.par.

(l) rec_unit.inc - determines the record unit.

\section{References}

[1] C. E. Brown, D. G. Ravenhall, Proc. R. Soc. London, Ser. A 208 (1951) 552.

[2] S. Fritzsche, C. Froese Fischer, G. Gaigalas, Comput. Phys. Comm. 148 (2002) $103-123$.

[3] C. Froese Fischer, G. Tachiev, G. Gaigalas, M. R. Godefroid, Comput. Phys. Comm. 176 (2007) $559-579$.

[4] P. Indelicato, J. P. Deslaux, Multi Configuration Dirac-Fock (2005). URL http://dirac.spectro.jussieu.fr/mcdf/mcdf_welcome/mcdf_homepage.html

[5] J. P. Deslaux, Comput. Phys. Comm. 9 (1975) 31-45.

[6] F. A. Parpia, C. Froese Fischer, I. P. Grant, Comput. Phys. Comm. 94 (1992) 249.

[7] P. Jönsson, X. He, C. Froese Fischer, I. P. Grant, Comput. Phys. Comm. 177 (2007) $597-622$.

[8] P. Jönsson, G. Gaigalas, J. Bieroń, C. Froese Fischer, I. P. Grant, Comput. Phys. Comm. 184 (2013) $2197-2203$.

[9] A. Hibbert, Comput. Phys. Comm. 9 (1975) $141-172$.

[10] W. Eissner, J. Phys. IV (France) 1 (1991) C1-3 - C1-13.

[11] R. D. Cowan, Theory of Atomic Structure and Spectra, University of California Press, Berkeley, 1981.

[12] A. Bar-Shalom, M. Klapisch, J. Oreg, J. Quant. Spectrosc. Rad. Transf. 71 (2001) 169 - 188.

[13] M. Ya. Amusia, L. V. Chernysheva, Computation of Atomic Process: A Handbook For ATOM programs, Institute of Physics Publishing, Bristol, 1997.

[14] M. F. Gu, Can. J. Phys. 86 (2008) $675-689$.

[15] M. J. Vilkas, K. Koc, Y. Ishikawa, Chem. Phys. Lett. 296 (1998) 68-76.

[16] V. A. Dzuba, V. V. Flambaum, M. G. Kozlov, Phys. Rev. A 54 (1996) 3948-59.

[17] I. M. Savukov, W. R. Johnson, Phys. Rev. A 65 (2002) 042503.

[18] V. A. Dzuba, Phys. Rev. A 71 (2005) 032512.

[19] V. A. Dzuba, V. V. Flambaum, Phys. Rev. A 75 (2007) 052504.

[20] S. G. Porsev, Yu. G. Rakhlina, M. G. Kozlov, Phys. Rev. A 60 (1999) 2781-5.

[21] S. G. Porsev, Yu. G. Rakhlina, M. G. Kozlov, J. Phys. B 32 (1999) 1113-1120.

[22] M. G. Kozlov, S. G. Porsev, Eur. Phys. J. D 5 (1999) 59-63.

[23] M. G. Kozlov, S. G. Porsev, W. R. Johnson, Phys. Rev. A 64 (2001) 052107. 
[24] S. G. Porsev, M. S. Safronova, M. G. Kozlov, Phys. Rev. Lett. 108 (2012) 173001.

[25] M. S. Safronova, S. G. Porsev, M. G. Kozlov, C. W. Clark, Phys. Rev. A 85 (2012) 052506.

[26] V. A. Dzuba, V. V. Flambaum, M. G. Kozlov, S. G. Porsev, Sov. Phys.-JETP 87 (1998) 885-90.

[27] M. S. Safronova, M. G. Kozlov, W. R. Johnson, D. Jiang, Phys. Rev. A 80 (2009) 012516.

[28] V. F. Brattsev, G. B. Deineka, I. I. Tupitsyn, Izv. Akad. Nauk SSSR, Ser. Fiz. 41 (1977) 2655, [Bull. Acad. Sci. USSR, Phys. Ser. 41 (1977) 173].

[29] P. Bogdanovich, G. Ẑukauskas, Sov. Phys. Collect. 23 (1983) 13.

[30] P. Bogdanovich, Lith. Phys. J. 31 (1991) 79.

[31] M. G. Kozlov, S. G. Porsev, V. V. Flambaum, J. Phys. B 29 (1996) 689-97.

[32] S. A. Kotochigova, I. I. Tupitsyn, J. Phys. B 20 (1987) 4759.

[33] E. R. Davidson, J. Comput. Phys. 17 (1975) 87-94.

[34] S. G. Porsev, M. G. Kozlov, Yu. G. Rakhlina, Pis'ma Zh. Eksp. Teor. Fiz. 72 (2000) 862-866, [JETP Lett. 72 595, (2000)].

[35] S. G. Porsev, M. G. Kozlov, Yu. G. Rakhlina, A. Derevianko, Phys. Rev. A 64 (2001) 012508.

[36] C. Cheng, X. L. Zhang, X. Gao, B. Qing, J. M. Li, J. Phys. B 43 (2010) 105001.

[37] Kramida, A., Ralchenko, Yu., Reader, J. and NIST ASD Team (2013). NIST Atomic Spectra Database (version 5.1). [http://www.nist.gov/pml/data/] National Institute of Standards and Technology, Gaithersburg, MD.

[38] C. Cheng, X. Gao, B. Qing, X. L. Zhang, J. M. Li, Chin. Phys. B 20 (2011) 033103.

[39] P. Jönsson, C. Froese Fischer, J. Phys. B 30 (1997) 5861.

[40] N. Rehbein, T. E. Mehlstäubler, J. Keupp, K. Moldenhauer, E. M. Rasel, W. Ertmer, A. Douillet, V. Michels, S. G. Porsev, A. Derevianko, C. Froese Fischer, G. I. Tachiev, V. G. Pal'chikov, Phys. Rev. A 76 (2007) 043406.

[41] A. Godone, C. Novero, Phys. Rev. A 45 (1992) 1717.

[42] D. Husain, J. Schifino, J. Chem. Soc. Faraday Trans. 280 (1984) 321.

[43] L. Liljeby, A. Lindgard, S. Mannervik, E. Veje, B. Jelencovic, Phys. Scr. 21 (1980) 805.

[44] L. Lundin, B. Engman, J. Hilke, I. Martinson, Phys. Scr. 8 (1973) 274. 